\title{
Thoracic endometriosis, a review
}

\begin{abstract}
Background: Thoracic endometriosis is the most frequent extra-pelvic location of endometrial lesions. Because thoracic endometriosis is an unusual and uncommon diagnosis in women, it is crucial that patients with catamenial chest pain and previous history of endometriosis undergo a thorough work-up. Due to the rarity of this disease a high index of clinical suspicion is imperative to make a diagnosis. Consequently, due to the multi-organ involvement of this disease a multi-disciplinary team is required for appropriate investigation, diagnosis, and treatment.
\end{abstract}

Presentation of the case: Patient is a 29-year-old Gravida 2, Para 0020 with a known history of pelvic endometriosis, confirmed by histopathology, was referred to our clinic for evaluation of her chronic pelvic pain, endometriosis, catamenial dyspnea, cyclic chest pain, dysmenorrhea, and menorrhagia.

The patient underwent robotic single-incision laparoscopic surgery (SILS) resection of endometriosis, ovarian cystectomy, lysis of adhesions, and cystoscopy by the Minimally Invasive Gynecologic Surgery (MIGS) team. Afterwards, the thoracic surgery team performed bilateral video-assisted laparoscopy surgery (VATS) with assistance from the MIGS to help identify suspicious lesions. Intraoperative evaluation revealed thoracic endometriosis confined to the pleura of the lungs and the diaphragm and were resected. Based on the clinical presentation and intraoperative findings the patient was diagnosed with thoracic endometriosis.

Conclusion: Thoracic endometriosis is an unusual, but relatively common diagnosis in women with catamenial chest pain, catamenial dyspnea, and previous history of confirmed endometriosis. Due to the impacts on patient quality of life and the potential complications of thoracic endometriosis, we find it imperative to investigate this patient population.

Keywords: catamenial hemoptysis, catamenial Pneumothorax, chronic pelvic pain, catamenial symptoms, cyclic chest pain, diaphragmatic endometriosis, Dienogest, dyspnea, elagolix (orilissa), endometriosis, endometriosis complications, endometriosis related pneumothorax, endometriosis surgery, minimally invasive gynecologic surgery, multidisciplinary team, orilissa, thoracic endometriosis, thoracic endometriosis syndrome, video-assisted laparoscopy surgery

Volume 10 Issue 5 - 2019

\author{
Shadi Rezai,' Alexander G Graves, ${ }^{2}$ \\ Cassandra E Henderson, ${ }^{3}$ Xiaoming Guan' \\ 'Division of Minimally Invasive Gynecologic Surgery, \\ Department of Obstetrics and Gynecology, Baylor College of \\ Medicine, USA \\ ${ }^{2}$ University of Queensland, Mayne Medical School,Australia \\ ${ }^{3}$ OB/GYN At Lake Success, One Hollow Lane, USA
}

Correspondence: Xiaoming Guan MD PhD, Division Chief and Fellowship Director, Division of Minimally Invasive Gynecologic Surgery, Department of Obstetrics and Gynecology, Baylor College of Medicine, 665I Main Street, 10th Floor, Houston, Texas, 77030, USA, Tel (832) 826-7464, Fax (832) 825-9349, Email xiaoming@bcm.edu

Received: July 21, 2019 | Published: September 24, 2019
Abbreviations: CPP, chronic pelvic pain; MIGS, invasive gynecologic surgery; TES, thoracic endometriosis syndrome; VATS, video-assisted laparoscopy surgery; SILS, single-incision laparoscopic surgery; OCPs, oral contraceptive pills; MRI, Magnetic resonance imaging; GnRH, gonadotropin-releasing hormone; BSO, bilateral salpingo-oophorectomy

\section{Background}

Cyclic chest pain with a catamenial relationship should increase suspicion of thoracic involvement with endometriosis. Similar to pelvic lesions these lesions are amenable to surgical resection. Due to the uncommon nature of thoracic involvement guidance and support from a gynecologic surgeon is a valuable resource to ensure complete and correct excision of suspected lesions.

\section{Presentation of the case}

Patient is a 29-year-old Gravida 2, Para 0020 with known history of endometriosis, who was referred to our clinic for further evaluation. The patient complained of chronic pelvic pain, endometriosis, catamenial hemoptysis (spitting blood with menses), cyclic chest pain, dysmenorrhea, and menorrhagia.
The patient's surgical history was significant for previous laparoscopic excisions/resection of endometriosis performed 2 years before presentation. Her family history was also significant for endometriosis.

On pelvic examination, there is bilateral adnexal tenderness with posterior fornix sweeping pain. On respiratory examination, there is catamenial shortness of breath and right sided chest pain with no hemoptysis.

Chest CT was normal with no visible lung nodules, implants, or pneumothoraxes. Transvaginal and transabdominal ultrasound showed an endometrioma or a hemorrhagic cyst within the right ovary measuring $3.4 \times 3.7 \mathrm{~cm}$ with the right ovary measuring $4.2 \times 4.4 \mathrm{~cm}$.

The patient underwent robotic single-incision laparoscopic surgery (SILS) resection of endometriosis, ovarian cystectomy, lysis of adhesions, and cystoscopy by Minimally Invasive Gynecologic Surgery (MIGS) team. After completion of the patient's pelvic surgery, the thoracic surgery team performed bilateral video-assisted laparoscopy surgery (VATS) with assistance from the MIGS to help identify suspicious lesions. Intraoperative evaluation revealed thoracic endometriosis confined to the pleura of the lungs and the 
diaphragm. These lesions were resected via electrocautery (Figures 1-3). No parenchymal lung lesions were identified. The patient had an uncomplicated recovery and postoperative phase. Upon discharge the patient was started on oral contraceptive pills (OCPs). Based on the clinical presentation and intraoperative findings the patient was diagnosed with thoracic endometriosis.

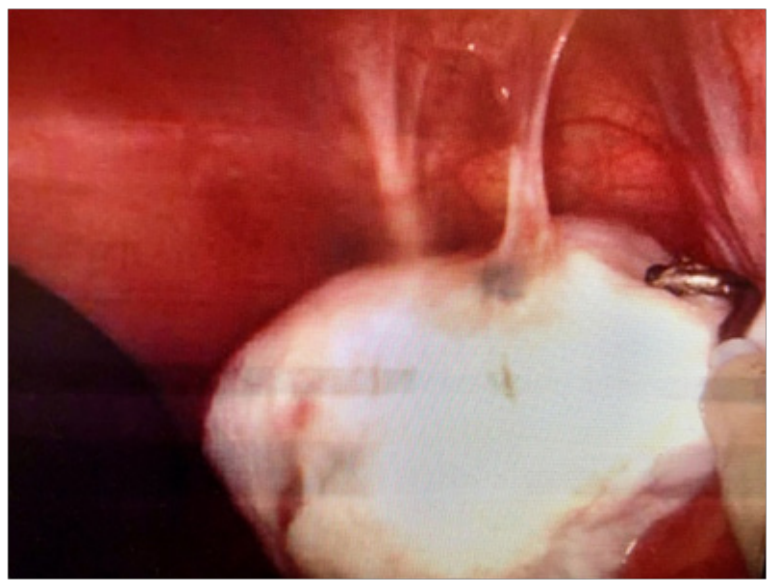

Figure I Endometriosis of the right ovary.

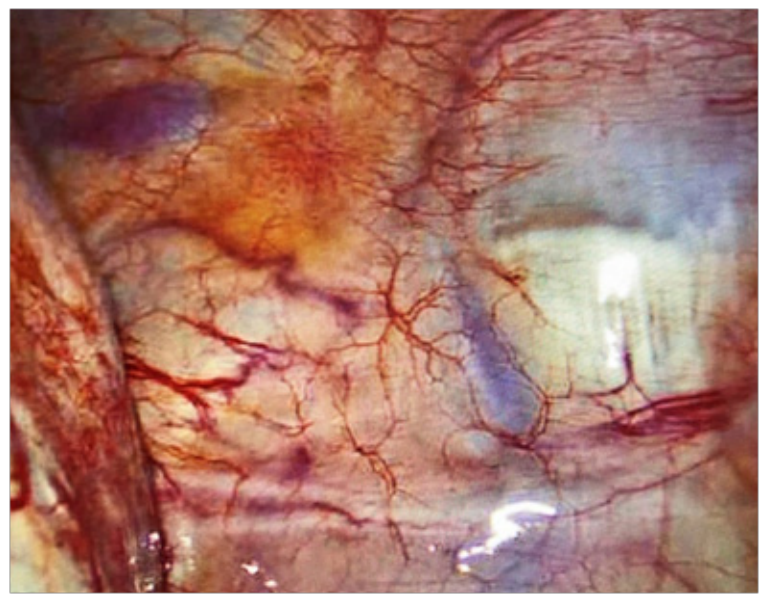

Figure 2 Lesions on the right pleural surface.

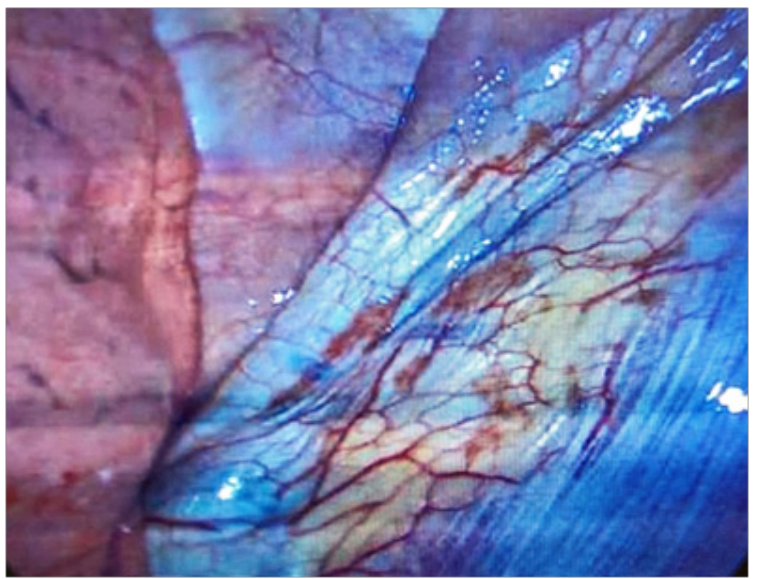

Figure 3 Lesions on the central tendon of the diaphragm (Diaphragmatic Endometriosis).

\section{Discussion}

Endometriosis is a benign condition characterized by uterine endometrial tissue outside of its normal location - most commonly involving the ovaries. ${ }^{1}$ In patients with extra-pelvic manifestations, thoracic involvement is most common, especially in patients with current pelvic disease. ${ }^{2,3}$ Laparoscopic resection is the gold standard for pelvic endometriosis, ${ }^{4}$ while evidence for surgical resection of thoracic endometriosis is limited due to the rarity of the disease. ${ }^{5}$ Therefore, coordinated care is of the utmost importance when treating this disease when it appears outside the pelvic cavity.

When discussing management of thoracic endometriosis there are important terms to define $e^{6}$ :

a. Thoracic endometriosis - endometrial tissue found in the thorax that is identified on histological specimens (hormone receptorpositive endometrial stroma and glands) obtained from chest tube aspirate, thoracotomy, or bronchoscopy.

b. Probable thoracic endometriosis - the identification of tissue within the thorax that is suggestive but not definitively diagnostic of endometrium (e.g., stroma only or hormone receptor-negative tissue).

c. Thoracic endometriosis syndrome-one or more clinical manifestations of thoracic involvement is present (e.g., pneumothorax, hemothorax, hemoptysis, chest pain) in association with menstruation but without histological confirmation.

d. Catamenial-the occurrence of symptoms or signs that bear a temporal relationship with menses.

There are several proposed mechanisms on how these lesions develop in the thorax. The three most popular theories are auto transplantation via retrograde menstruation, micro-embolization/ metastasis, and coelomic metaplasia with auto transplantation being the most popular theory. ${ }^{7}$

The autotransplantation via retrograde menstruation theory proposes that ectopic endometrium may migrate through diaphragmatic defects. ${ }^{8,9}$ However, three different observations weaken this theory. First, diaphragmatic defects are uncommon, and the overwhelming majority of thoracic endometriosis are predominantly right-sided ( $\sim 95 \%$ of cases) ${ }^{3}$ if defects were truly portals of entry for endometrial tissue, the disease would be expected to be bilateral in distribution. Second, there can be a recurrence of symptoms even after a diaphragmatic repair and/or hysterectomy. ${ }^{10}$ Finally, ectopic endometrial tissue differs significantly from eutopic endometrium in clonality, enzymatic activity, protein expression, and histologic properties..$^{11,12}$

The micro-embolization/metastasis theory suggests metastatic spread of endometrial tissue through the venous or the lymphatic system to the lungs. ${ }^{13,14}$ Endometrial foci in tissue remote from the pelvis and thorax (e.g., brain, knee, and eye) support the "metastatic theory." However, in opposition to this theory, micro-embolization would be expected to affect both hemithoraces approximately equally, whereas thoracic endometriosis is found overwhelmingly in the right hemothorax. ${ }^{14}$

The coelomic metaplasia theory suggests there is a transformation of pluripotent cells to differentiated endometrium. In support of this theory, pluripotent cells have been identified in uterine endometrium 
and a case of endometriosis has been reported in a 20-year-old woman with congenital agenesis of uterus, cervix, vagina, and fallopian tubes. ${ }^{15}$

The etiology is likely to be multifactorial given that not one theory explains the pathogenesis of endometriosis, but rather a conglomeration of the three theories does.

\section{Clinical presentation}

There are four main clinical presentations with thoracic endometriosis: catamenial pneumothorax, catamenial hemothorax, catamenial hemoptysis, and pulmonary nodules. The most common presentation ( 70 to $73 \%$ ) of thoracic endometriosis is catamenial Pneumothorax. ${ }^{5}$ A smaller proportion (approximately 15\%), has catamenial pneumothorax due to probable thoracic involvement, and 10 percent or less have pneumothorax that has no temporal relationship with menses (non-catamenial pneumothorax). ${ }^{5}$ Patients with endometriosis-related non-catamenial pneumothorax are typically identified during surgery for recurrent pneumothorax.

Catamenial hemothorax is the next most common manifestation of thoracic endometriosis. Hemorrhage into the pleural space occurs in less than 15 percent of patients with thoracic endometriosis. ${ }^{3,14}$ Compared with endometriosis-associated pneumothorax, endometriosis-associated hemothorax is typically associated with extensive pleural endometrial implants.

Catamenial hemoptysis occurs in less than 14 percent of patients and is typically due to parenchymal or endobronchial endometriosis. ${ }^{3,16}$ The mean age of patients presenting with hemoptysis (26 to 29 years) appears to be lower than patients with other manifestations of thoracic endometriosis ( 34 years). ${ }^{3,17}$ Bleeding is usually catamenial, minor, and right-sided. The least common manifestation of thoracic endometriosis is pulmonary nodules. It can present as solitary or multiple pulmonary nodules ( 2 to $6 \%$ of all patients). Nodules can be incidental or symptomatic (e.g., hemoptysis or chest pain), vary in size $(0.5$ to $3 \mathrm{~cm})$ and are typically right-sided. . $^{3,18,19}$

In patients with early disease that is minimally and mainly confined to the diaphragm the above four clinical presentations may not be appreciable In cases where the endometriosis is confined to the diaphragm there can be associated pain in the ipsilateral chest, shoulder, arm, and neck and rarely can lead to diaphragmatic rupture and pneumoperitoneum. ${ }^{20}$

\section{Diagnosis}

As there is no gold standard diagnostic method, test, or imaging for thoracic endometriosis the diagnosis is usually on a clinical ground. Since this is a rare disease and there is a wide spectrum of presentation, a high index of clinical suspicion is key to recognize this disease. When a premenopausal woman presents with catamenial dyspnea, cough, and chest pain it is crucial the examining physician assess the possibility of thoracic involvement of endometriosis and make it component of the differential diagnosis. Symptoms have a catamenial pattern, occurring between 24 hours before and 72 hours after the onset of menses,$^{18}$ and typically recurring. ${ }^{5}$ Catamenial pneumothorax is defined by at least two episodes of pneumothorax occurring during this time interval. ${ }^{5}$ The right-side predominance of symptoms represents a diagnostic clue. A thorough and targeted physical examination may reveal diminished or absent breath sounds, dullness to percussion and decreased chest expansion, which could suggest a pleural effusion. Furthermore, decreased breath sounds, hyperresonance, and decrease chest expansion suggest a pneumothorax.

Imaging is important to evaluate any history and focal exam findings suggesting thoracic pathology. A chest x-ray can help elucidate a pneumothorax, pleural effusions, or pulmonary nodules. $\mathrm{CT}$ can further assess if there is a pneumothorax, pleural effusions, or pulmonary nodule if the chest $\mathrm{x}$-ray was inconclusive. However, CT is considered the first-line imagining, as it can rule out other diagnoses and map the lesions for surgery if necessary. ${ }^{21}$ Unfortunately, CT imagining is poorly specific; therefore, its main role is to rule out other pulmonary diseases.

Magnetic resonance imaging (MRI) is an alternative to CT imaging. The lack of spatial resolution, as compared to CT, is compensated by a higher contrast resolution and allows a better depiction of hemorrhagic lesions. There are two drawbacks with the use of MRI. First, a single breath hold is easily achievable with a CT, but this becomes an impossible feat with the MRI. Second, the presence of air close to the diaphragm may make it difficult to distinguish true lesions from artefacts. Overall, MRI may be superior to CT only because of less radiation exposure. ${ }^{22}$

There is a limited role for bronchoscopy in diagnosis because most pathologic features are located in the peripheral lung. There have been case reports of success with bronchoscopy, but there are few cases presented in the literature..$^{23}$

\section{Treatment}

Despite the lack of randomized studies, there is disagreement in the literature regarding first line therapy as whether it should be surgical or medical therapy first. Some experts believe that the majority patients with thoracic endometriosis should be treated with hormone suppressive therapy as opposed to surgery as first line therapy. Data from observational studies support a reduction in the rate of recurrent pneumothorax and hemothorax with hormonal agents alone. ${ }^{24}$ Whereas, this is support for surgical management as first line therapy when there is no/low response to hormonal therapy. ${ }^{25}$

In regard to medical therapy, Dienogest, a progestogenic drug with moderate estrogen suppression, anti-inflammatory, antiproliferative, and anti-angiogenic properties, is a new treatment available for initial management of thoracic endometriosis. ${ }^{17}$

Typically, gonadotropin-releasing hormone (GnRH) analogs are the first-line agents because they are highly effective at suppressing ovarian hormone production and inhibiting the growth of endometrial tissue. ${ }^{3,5,26,27}$ However, prolonged therapy with GnRH analogs is often complicated by intolerance from the acute symptoms of menopause and the risk of osteoporosis. Consequently, the decision to use GnRH analogs is dependent upon weighing the benefits of reduced recurrence against the adverse effects in the context of patient preferences regarding the temporary induction of menopause and inability to become pregnant.

For those not willing to become menopausal or who have a contraindication to GnRH analogs, oral contraceptives or progestins, and less commonly, danazol or aromatase inhibitors, are alternatives. The advantage of oral contraceptive agents is that endometrial implant stimulation is low grade and withdrawal bleeding can be avoided, if necessary, by the administration of continuous therapy. All hormonal agents should be stopped in those who cannot tolerate the side effects and in those who have a desire to become pregnant. Recurrence 
rates are high particularly when hormonal suppressive therapy is not administered. However, some patients develop recurrence despite use of hormonal therapy.

In patients who are unable to undergo medical management or who have refractory disease, surgery may be a secondary treatment option. However, the optimal therapy for patients with refractory disease is unknown.

If the patient and surgeon agree on surgery as first line, current available options are: pleurectomy and repeat pleurodesis for recalcitrant pneumothorax or hemothorax and hysterectomy with or without bilateral salpingo-oophorectomy (BSO). In the thorax, all surgical techniques should be directed at pleural adhesion to the thoracic wall to remove any potential space for pathologic features. ${ }^{28}$ Although, these surgical options cause infertility, surgical management should always be discussed.

Once the patient has been treated for thoracic endometriosis with or without pelvic involvement, she should be followed by her gynecologist as well as her pulmonary surgeon at regular intervals to assess reoccurrence until it is deemed, she has reached a time of disease-free progression.

\section{Conclusion}

Due to the numerous anatomic locations of endometriosis and the inherent complexity of the disease we find it is imperative that a multi-disciplinary team delivers coordinated care to achieve the best outcome for the patient. We have found the two-team approach to be the safest and most effective approach to treating patients with thoracic endometriosis, consisting of a thoracic surgeon and gynecologist. While the thoracic surgeon is operating in the thorax, the gynecologist is present to assist and advise on any suspicious lesions that may require resection.

In terms of diagnosis, specialists must have a high index of suspicion for thoracic endometriosis when a patient presents with catamenial chest symptoms on a background of pelvic endometriosis. In these patients, further history, focused examination, and investigations should be initiated, including chest imaging (CT or MRI) to rule out another chest pathology. Video assisted thoracic surgery (VATS) should also be discussed with the patient as a mode of definitive diagnosis.

First line treatment of thoracic endometriosis is still debated within the literature,. Ultimately, we find that the direction of therapy, whether it be non-invasive or invasive measures, is strongly guided by patient preference. As we see patients in our surgical gynecology clinic, the vast majority of them have failed medical hormonal therapy or it has not been sufficient to reduce their symptoms. We offer minimally surgery to these patients as a more definitive treatment. The risks and benefits are thoroughly discussed with the patient with adequate insight into how each treatment option can affect their personal lives and their reproductive health.

\section{Acknowledgments}

Dr. Xiaoming Guan is a speaker for Applied Medical, Rancho Santa Margarita, California.

\section{Funding}

None.

\section{Conflicts of interest}

Authors did not report any potential conflicts of interests.

\section{References}

1. Giudice LC, Kao LC, Endometriosis. Lancet. 2004;364(9447):1789-1799.

2. Bagan P, Berna P, Assouad J, et al. Value of cancer antigen 125 for diagnosis of pleural endometriosis in females with recurrent pneumothorax. Eur Respir J. 2008;31(1):140-142.

3. Joseph J, Sahn SA. Thoracic endometriosis syndrome: new observations from an analysis of 110 cases. Am J Med. 1996;100(2):164-170.

4. Scarselli G, Rizzello F, Cammilli F, et al. Diagnosis and treatment of endometriosis. a review. Minerva Ginecol. 2005;57(1):55-78.

5. Alifano M, Jablonski C, Kadiri H, et al. Catamenial and non-catamenial, endometriosis-related or non-endometriosis-related pneumothorax referred for surgery. Am J Respir Crit Care Med. 2007;176(10):1048-1053.

6. Joseph-Vempilly J, Courtney Broaddus V, Finlay G. Thoracic endometriosis: pathogenesis, epidemiology, and pathology. UpToDate. 2019.

7. Vinatier D, Orazi G, Cosson M, et al. Theories of endometriosis. Eur $J$ Obstet Gynecol Reprod Biol. 2001;96(1):21-34.

8. Cooper MJ, Russell P, Gallagher PJ. Diaphragmatic endometriosis, Med J Aust. 1999;171(3):142-143.

9. Cowl CT, Dunn WF, Deschamps C. Visualization of diaphragmatic fenestration associated with catamenial pneumothorax. Ann Thorac Surg. 1999;68(4):1413-1414.

10. Joseph J, Reed CE, Sahn SA. Thoracic endometriosis. Recurrence following hysterectomy with bilateral salpingo-oophorectomy and successful treatment with talc pleurodesis. Chest. 1994;106(6):1894-1896.

11. Redwine DB. Was Sampson wrong? Fertil Steril. 2002;78(4):686-693.

12. Bouquet De Jolinière J, Ayoubi JM, Gianaroli L, et al. Endometriosis: a new cellular and molecular genetic approach for understanding the pathogenesis and evolutivity. Front Surg. 2014;1:16.

13. Duke R, Fawcett P, Booth J. Recurrent subarachnoid hemorrhage due to endometriosis. Neurology. 1995;45(5):1000-1002.

14. Channabasavaiah AD, Joseph JV. Thoracic endometriosis: revisiting the association between clinical presentation and thoracic pathology based on thoracoscopic findings in 110 patients. Medicine (Baltimore). 2010; 89(3):183-188.

15. Mok-Lin EY, Wolfberg A, Hollinquist H, et al. Endometriosis in a patient with Mayer-Rokitansky-Küster-Hauser syndrome and complete uterine agenesis: evidence to support the theory of coelomic metaplasia. $J$ Pediatr Adolesc Gynecol. 2010; 23(1):e35-e37.

16. Nezhat C, Main J, Paka C, et al. Multidisciplinary treatment for thoracic and abdominopelvic endometriosis. JSLS. 2014;18(3).

17. Fukuda S, Hirata T, Neriishi K, et al. Thoracic endometriosis syndrome: Comparison between catamenial pneumothorax or endometriosis-related pneumothorax and catamenial hemoptysis. Eur J Obstet Gynecol Reprod Biol. 2018;225:118-123.

18. Alifano M, Trisolini R, Cancellieri A, et al., Thoracic endometriosis: current knowledge, Ann Thorac Surg. 2006;81(2):761-769.

19. Flieder DB, Moran CA, Travis WD, et al. Pleuro-pulmonary endometriosis and pulmonary ectopic deciduosis: a clinicopathologic and immunohistochemical study of 10 cases with emphasis on diagnostic pitfalls. Hum Pathol. 1998;29(12):1495-503.

20. Redwine DB. Diaphragmatic endometriosis: diagnosis, surgical 
management, and long-term results of treatment. Fertil Steril. 2002;77(2):288-296

21. Rousset P, Rousset-Jablonski C, Alifano M, et al. Thoracic endometriosis syndrome: CT and MRI features. Clin Radiol. 2014;69(3):323-330.

22. Cassina PC, Hauser M, Kacl G, et al. Catamenial hemoptysis. diagnosis with MRI. Chest. 1997;111(5):1447-1450.

23. Shiota Y, Umemura S, Arikita H, et al. A case of parenchymal pulmonary endometriosis, diagnosed by cytologic examination of bronchial washing. Respiration. 2001;68(4):439.

24. Römer T. Long-term treatment of endometriosis with dienogest: retrospective analysis of efficacy and safety in clinical practice. Arch Gynecol Obstet. 2018;298(4):747-753.
25. Marshall MB, Ahmed Z, Kucharczuk JC, et al. Catamenial pneumothorax: optimal hormonal and surgical management. Eur J Cardiothorac Surg. 2005;27(4):662-666.

26. Korom S, Canyurt H, Missbach A, et al. Catamenial pneumothorax revisited: clinical approach and systematic review of the literature. $J$ Thorac Cardiovasc Surg. 2004;128(4):502-508.

27. Akal M, Kara M. Nonsurgical treatment of a catamenial pneumothorax with a Gn-RH analogue. Respiration. 2002;69(3):275-276.

28. Härkki P, Jokinen JJ, Salo JA, et al. Menstruation-related spontaneous pneumothorax and diaphragmatic endometriosis. Acta Obstet Gynecol Scand. 2010;89(9):1192-1196. 